during embolization and thus may reduce procedural complications.

Material and Methods 3D AVM surface models were extracted from multimodal image data $3 \mathrm{D}$ digital subtraction image and MRA). For the virtual embolisation, all feeders of each AVM were identified and blocked separately. For $\mathrm{n}$ feeder arteries each blocked or unblocked, a total amount of $n^{2}$ blood flow simulations via computational fluid dynamics (CFD) was carried out. A custom visualization and exploration tool was developed using the Game Engine Unity where blood flow was illustrated via millions of small particles based on the CFD results.

Results The virtual embolisation software prototype allows the clinical expert to explore patient specific AVM models in 3D. Furthermore, the user can select and deselect feeder arteries to block (i.e. to embolize) or unblock them, respectively. Thus, the effects of an individual embolization strategy can be explored by analyzing the subsequent blood flow as well as by interpreting the resulting pressure gradients.
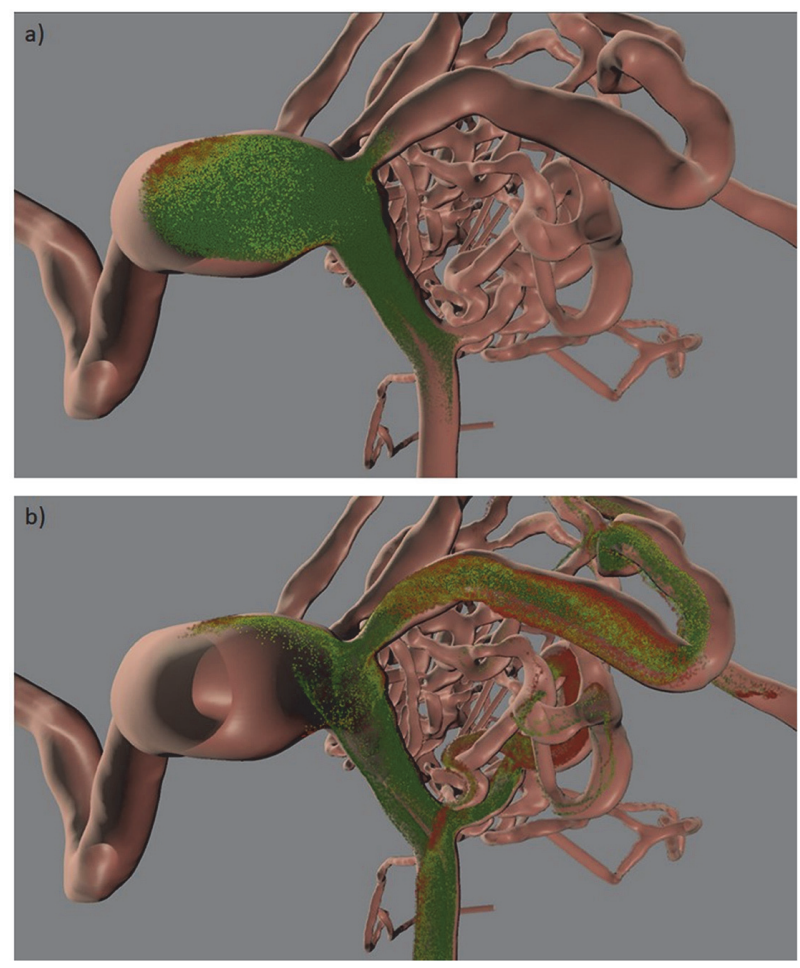

Abstract E-143 Figure 1 Depiction of the introduced AVM blood flow visualization. The user chooses a starting point of blood flow particles in the internal carotid artery and can follow the spread of particles along the feeder arteries to get an impression how the different feeders contribute to the blood supply of the nidus

Conclusion Because the success and safety of AVM treatment is a matter of hemodynamic balance until the draining veins are occluded by the embolization material, virtual AVM embolization is a powerful tool to simulate and explore the hemodynamic changes that occur at different embolization steps prior to AVM treatment.

Disclosures D. Behme: None. U. Sprengel: None. J. Stahl: None. P. Saalfeld: None. B. Behrendt: None. M. Thormann: None. A. Mpotsaris: None. B. Preim: None. P. Berg: None. S. Saalfeld: None.

\section{E-144 THE PRESENCE OF CEREBRAL EDEMA IN ADDITION TO RETROGRADE LEPTOMENINGEAL VENOUS DRAINAGE IN CRANIAL DURAL ARTERIOVENOUS FISTULAS IS AN INDICATOR OF CLINICAL SEVERITY}

I Nakagawa*, H Park, M Kotsugi, S Yokoyama, T Furuta, K Nakase, A Okamoto, S Yamada, H Nakase. Neurosurgery, Nara Medical University, Kashihara, Japan

\subsection{6/neurintsurg-2021-SNIS.239}

Purpose Retrograde leptomeningeal venous drainage (RLVD) in dural arteriovenous fistulas (DAVFs) is associated with intracerebral hemorrhage and nonhemorrhagic neurological deficits or death. Although angiographic evidence of RLVD is a definite indication for treatment, the addition of venous congestion to RLVD may cause severe clinical symptoms. In this study, we assessed parenchymal cerebral blood volume (PBV) to evaluate whether the presence of cerebral edema in addition to RLVD predicts the clinical severity in DAVFs.

Methods We retrospectively identified 53 patients who had angiographic evidence of RLVD and received treatment. The presence of cerebral edema was defined as cortical hyperintensity in FLAIR image. The PBV was obtained from rotational angiography and analyzed by workstation (syngo XWP vD $10 \mathrm{E})$.

Results In the cerebral edema group $(n=17)$, cerebral microbleeds, venous congestion ratio, PBV ratio, and shunt circulation time (SCT) were significantly increased $(p<0.001)$, and modified rankin scale $>2$ at discharge was significantly higher $(p=0.014)$. The preoperative PBV ratio was significantly correlated with the angiographic SCT and venous congestion ratios $(\mathrm{p}<0.001)$.

Conclusion Evaluation of PBV is useful for assessing focal venous congestion, and the presence of cerebral edema in addition to RLVD in DAVF can be an indicator of clinical severity.

Disclosures I. Nakagawa: None. H. Park: None. M. Kotsugi: None. S. Yokoyama: None. T. Furuta: None. K. Nakase: None. A. Okamoto: None. S. Yamada: None. H. Nakase: None.

\section{E-145 SAFETY OF PEDIATRIC CEREBRAL ANGIOGRAPHY}

${ }^{1} \mathrm{D}$ Lauzier*, ${ }^{2} \mathrm{~J}$ Osbun, ${ }^{2} \mathrm{~A}$ Chatterjee, ${ }^{3} \mathrm{C}$ Moran, ${ }^{2} \mathrm{~A}$ Kansagra. ${ }^{1}$ Mallinckrodt Institute of Radiology, Washington University School of Medicine, St. Louis, MO; ${ }^{2}$ Mallinckrodt Institute of Radiology, Department of Neurological Surgery, Department of Neurology, Washington University School of Medicine, St. Louis, MO; ${ }^{3}$ Mallinckrodt Institute of Radiology, Department of Neurological Surgery, Washington University School of Medicine, St. Louis, MO

10.1136/neurintsurg-2021-SNIS.240

Introduction/Purpose Catheter-based cerebral angiography is commonly used for neurovascular diagnosis in children. In this work, we aim to quantify the complication rate of cerebral angiography in children, characterize these complications, and identify risk factors for complications.

Materials and Methods Relevant clinical data were retrospectively obtained for 587 consecutive cerebral angiography procedures performed in 390 children from March 2002 to March 2020. Complications were categorized as neurologic or non-neurologic, and severity was graded using a standard schema. Incidences of complications are reported as point estimates. Associations between risk factors and complications were characterized in univariate analysis using the two-tailed 